PROGRAM DIRECTOR'S NOTEBOOK

\title{
'Blueprint' for designing a curriculum for intern and residency programs
}

\author{
DANIEL D. TOMASZEWSKI
}

Postgraduate training faces new challenges as physicians become more specialized, the lay population becomes more sophisticated, and those resources necessary to train physicians become scarcer. A nine-step model is presented for developing a curriculum that will prepare healthcare professionals to practice in this changing socioeconomic milieu.

(Key words: Postgraduate training, curriculum development, education, intern and residency programs)

The growth of education as a social science in this century has led educators and trainers away from the traditional model of apprentice training, developed by 12 th century European guilds, to a curriculum-based model. This model has altered the perception of how physicians should be trained. More than just a schedule of places where the apprentice physician spends time under the guidance of a master, the curriculum has become an explicit, objective-based document. Curriculum-based training specifies the desired outcomes of the training experience by defining optimal performance as well as minimal competencies and expectations.

Social science has not been the only determinant in the development of this model. Recent profound social and economic changes in

$\overline{\mathrm{Mr} \text { Tomaszewski is associate director of medical educa- }}$ tion, Detroit Osteopathic and Bi-County Hospitals, Highland Park, Mich.

Reprint requests to Daniel D. Tomaszewski, Detroit Osteopathic Hospital, c/o Medical Education, 12523 Third Ave, Highland Park, MI 48203-3294.
Western societies have made the adaption of curriculum-based training a necessity, rather than a luxury, in medicine. Within the healthcare system, increasing specialization, and hence differences, exist among physician roles. As physician roles change, so do relationships and behaviors with other healthcare professionals. Biotechnology has compounded physicians' value- and ethical-decision-making responsibilities. With shrinking resources and exponential increases in information, more efficient, effective use of limited education resources-time, finances, and personnel-has become imperative. Furthermore, the entire healthcare system must now operate within an increasingly knowledgeable and assertive lay population. This vast social ecology has demystified medicine and increased the healthcare system's accountability to other areas of society.

\section{Curriculum uses}

Curriculum-based postgraduate training enhances the abilities of training institutions, trainers, interns, and residents to meet these challenges. Developing a curriculum includes not only an end product-a document-but it also involves a dynamic process that entails active, and often heated, discussion concerning what should be taught and how.

Specifically, curriculum development may be used to accomplish the following:

- Provide a systematic consideration of the physician's role.

- Assist in defining accountability for physician practices and policies.

- Guide teaching and instructional activities. 
- Provide a base for feedback from trainees regarding level of preparation as well as a base for program and trainee evaluation.

- Establish a guide for audits and protocols.

- Describe the training program.

- Raise the physician's awareness of the educator's role.

- Institute a common mission and goals for faculty development.

With these objectives in mind, I propose the following nine-step model for curriculum development in a training hospital:

\section{Organize curriculum task force.}

The program director is responsible for curriculum development. This development is actually a long-term, ongoing process. A task force may spend months on a curriculum development project. This process may need to be repeated as often as evaluation of the program deems changes are necessary.

The program director quickly realizes that a successful project is the result of key individuals - from within and outside the program faculty-who can work together throughout the development process. The program director can significantly improve the chances for a successful project by involving the entire medical staff and faculty in selecting task force members. Nonphysician healthcare professionals, particularly behavioral scientists interested in the psychosocial aspects of training and in evaluation, may be helpful to the overall process. Such individuals should be incorporated during the initial stages.

Providing a clear description of the process and roles assigned to interested individuals will facilitate the desired outcome-a curriculum document endorsed by the entire program faculty. Similarly, building and maintaining continued faculty support means emphasizing those valuable elements in the existing program. The program director can then build a case for change from task force members' shared concerns.

\section{Adapt a curriculum development model.}

Once selected, the members of the task force should establish a curriculum development model that they intend to follow. Group lead- ership and tentative timetables should be established for the curriculum's various phases.

\section{Describe the specialist physician's role.}

This step involves systematically identifying what specialty physicians actually do. Often, the best way to describe the specialty practice is to survey graduates of the training institution who have been practicing for a number of years. It also entails identifying those behaviors peculiar to the specialty, as well as those that, while not essential, are important dimensions of that specialty practice.

Other questions need to be asked at this stage: Where will these graduates practice? What is the future of the specialty?

\section{Identify the education and training goals.}

Goal statements are based on those actualand desired-behaviors that trainers would like trainees to exhibit in their future practice of medicine. These goals reflect the unique characteristics and values of the specialty and should encompass all of the important aspects of a specialty practice. Major goals should be limited to four or five.

\section{Develop a curriculum content outline.}

The content outline should list major areas of measurable physician behavior. The outline may first cover more general performance areas (physical examination, for example) before detailing more specific areas, such as problems of the cardiovascular system.

\section{Specify objectives for each content area.}

The content outline becomes the foundation for developing specific performance objectives, criteria, and timetables for each content area. Objectives provide the behavior standards by which all trainee performance is measured. Objectives describe precise, measurable behaviors, which can be evaluated. Both trainer and trainee know these objectives at the beginning of the training experience.

\section{Develop faculty consensus on all objectives.}

Here, again, the program director's ability to lead the program faculty from a shared concern to actually reaching a consensus is criti- 
cal. For every objective, each faculty member's expert opinion should be solicited and acknowledged.

\section{Submit curriculum draft document to entire program faculty.}

The entire program faculty reviews the draft document and submits proposed changes to the task force.

\section{Faculty approves revised curriculum.}

The approved document becomes the basis for program, faculty, and trainee evaluation.

\section{Summary}

Successful curriculum development entails a task force's ability to build on those valuable elements from the existing program. This new program should reflect those characteristics and traits inherent to that particular specialty. The curriculum must be able to accommodate the changing social and economic environment in which physicians find themselves practicing medicine. As such, curriculum development is an ongoing process.

\section{Bibliography}

Barker LR: Curriculum for ambulatory care training in medical residency. J Gen Intern Med 1990;5(Jan/Feb suppl):S3-S14.

Jensen NM, Dirkx JM: A Curriculum for Internal Medicine Residency: The University of Wisconsin Program. Philadelphia, $\mathrm{Pa}$, American College of Physicians, 1989, 174 pp.

Nelson RL, McCaffrey LA, Nobrega FT, et al: Altering residency curriculum in response to a changing practice environment. Uses of the Mayo internal medicine alumni survey. Mayo Clin Proc 1990;65:809-817.

Vontver LA: A use of instructional objectives to increase learning efficiency. $J$ Med Educ 1974;49:453-454.

Wood DL: Redefining residency goals, objectives and evaluation. The DO 1990;31(12):79-82. 